\title{
High-Performance Estimation of Lead Ion Concentration Using Smartphone-Based Colorimetric Analysis and a Machine Learning Approach
}

Samira Sajed, Mohammadreza Kolahdouz,* Mohammad Amin Sadeghi, and Seyedeh Fatemeh Razavi

Cite This: ACS Omega 2020, 5, 27675-27684

Read Online

ABSTRACT: Traditional methods for detection of lead ions in water samples are costly and time-consuming. In this work, an accurate smartphone-based colorimetric sensor was developed utilizing a novel machine learning algorithm. In the presence of $\mathrm{Pb}^{2+}$ ions in the solution of specifically functionalized gold nanoparticles, the color of solution turns from red to purple. Indeed, the color variation of the solution is proportional to $\mathrm{Pb}^{2+}$ concentration. The smartphone camera captures the corresponding color change, and the image is processed by an efficient artificial intelligence protocol. The nonlinear regression approach was used for concentration estimation, in which the parameters of the proposed model are obtained using a new feature extraction

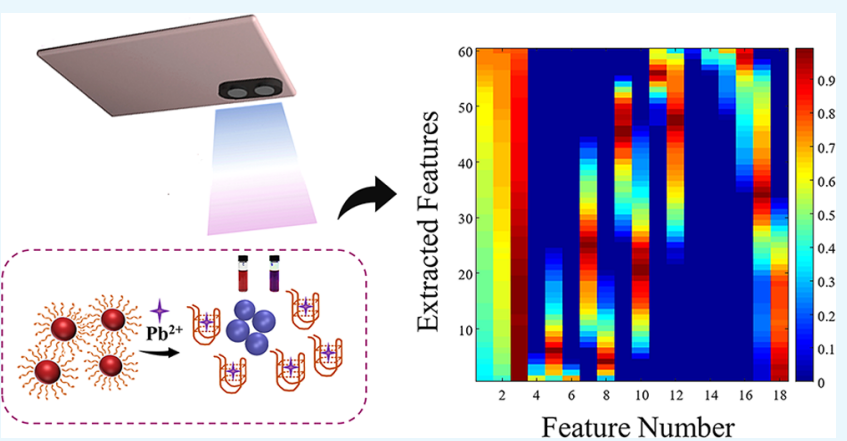
algorithm. In prediction of $\mathrm{Pb}^{2+}$ concentration, the average absolute error and root-mean-square error were 0.094 and 0.124 , respectively. The influence of $\mathrm{pH}$ of the medium, temperature, oligonucleotide concentration, and reaction time on the performance of the proposed sensor was carefully investigated and understood to achieve the best sensor response. This novel sensor exhibited good linearity for the detection of $\mathrm{Pb}^{2+}$ in the concentration range of $0.5-2000 \mathrm{ppb}$ with a detection limit of $0.5 \mathrm{ppb}$.

\section{INTRODUCTION}

Lead is an abundant toxic heavy metal, which is well known to be nondegradable. Even a tiny amount of lead in the human food chain will cause irreversible damage to the human nervous system, kidneys, liver, and so on. ${ }^{1,2}$ Since identification of serious destructive effects of lead on human health was carried out in the 1970s, a variety of methods have been developed for monitoring of lead amounts in the real and environmental samples. The U.S. Environmental Protection Agency (EPA) set the lead toxic level in the drinking water as $72.5 \mathrm{nM}(15 \mathrm{ppb}) .^{3}$

Several conventional techniques are available for the determination of lead, including atomic absorption/emission spectrometry, ${ }^{4,5}$ inductive coupled plasms mass spectrometry (ICP-MS), ${ }^{6}$ high-performance liquid chromatography, ${ }^{7}$ and $\mathrm{X}$-ray fluorescence spectrometry. ${ }^{8,9}$ These methods are sensitive but have significant drawbacks, such as timeconsuming analysis, complex sample preparation process, well-trained personnel, and costly instruments.

Recent advances in optical, chemical, and biological sensors have shown great promise for a more convenient alternative method for lead detection, but most of them are not applicable in resource-limited environments. ${ }^{10,11}$ The nanoparticles are being developed in different forms as important receptors for heavy-metal ions. ${ }^{12-14}$ Son and co-workers synthesized a magnetic silica nanoparticle for $\mathrm{Pb}^{2+}$ detection. The surface- functionalized probe acts as a new type of synthetic fluorochromogenic sensor for imaging $\mathrm{Pb}^{2+}$ ions in living cells. $^{15}$

Among the several metal nanoparticles, gold nanoparticles (GNPs) have become more attractive because of visible region characteristics, biocompatibility, chemical stability, and water solubility. ${ }^{16,17}$

The optical properties of GNPs depend on their size, shape, surface structure, aggregation state, and dielectric medium. ${ }^{18}$ The GNP interaction with the target analyte and the aggregation rate increase at higher GNP concentrations. Therefore, the higher the concentration of GNPs, the stronger the nanoprobe will be. However, the small changes in solution color will not be observed at high GNP concentrations because of the stronger background signal. However, lower GNP concentrations will reduce the limit of detection (LOD) because the number of nanoprobes has been reduced. As a result, the best LOD occurs at intermediate GNP concen-

Received: August 31, 2020

Accepted: October 5, 2020

Published: October 16, 2020 

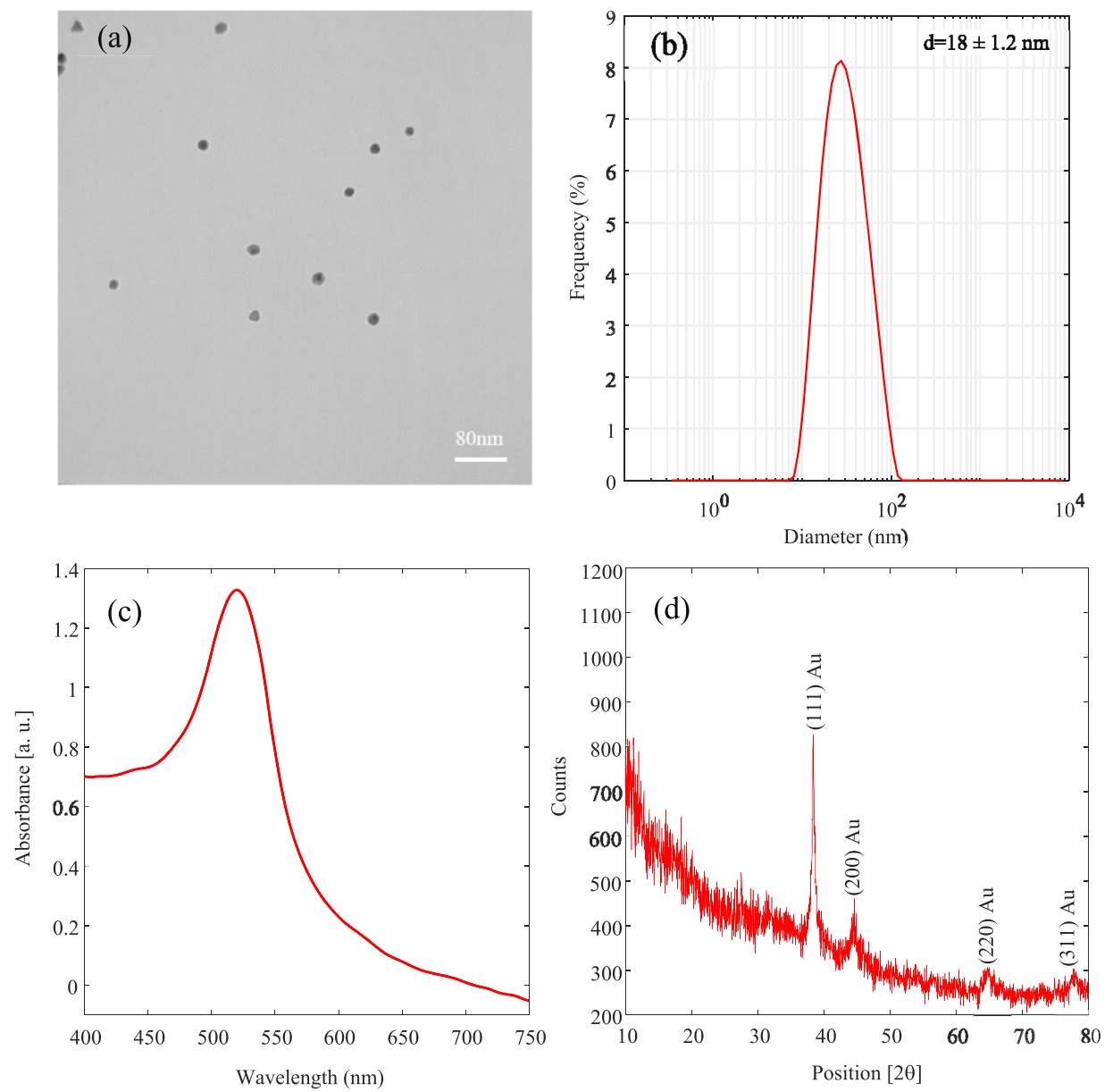

Figure 1. (a) TEM image, (b) particle size and Gaussian size distribution, (c) UV-visible absorption spectrum, and (d) X-ray diffraction of synthesized GNPs.

trations because of a balance between the background signal and aggregation rate. ${ }^{19}$

In the case of heavy-metal ion detection, the presence of target ions mainly aggregates the GNPs, which in turn, leads to a red shift of localized surface plasmon resonance (LSPR) peak and the corresponding color change. ${ }^{20}$ According to the reported studies, the GNP's surface is functionalized by aptamers and DNases, ${ }^{21,22}$ molecules containing carboxyl group, sulfhydryl groups, and nitrogen for the detection of lead ions. ${ }^{23-26} \mathrm{Xu}$ et al. functionalized GNP's surface with a guanine-rich oligonucleotide for lead ion detection. This oligonucleotide prevents $\mathrm{NaCl}$-induced aggregation of GNPs. On addition of $\mathrm{Pb}^{2+}$ ions, oligonucleotides release into the solution, and the color of the solution turns from red to blue. Their method was a simple, rapid, and selective colorimetric assay with the lowest detectable concentration of $20 \mathrm{nM}^{21}$

The colorimetric systems accompanied by smartphone platforms have demonstrated great potential as a portable, sensitive, and cost-effective approach for analysis of different analytes. ${ }^{27,28}$ Nowadays, smartphones are ubiquitous and host a variety of advanced sensors, especially, powerful camera and wireless connectivity technology. So far, the smartphone platforms were used for the detection of heavy-metal ions, ${ }^{29-31}$ molecules, ${ }^{32}$ cells, and biomarkers. ${ }^{3-35}$ In general, a simple apparatus should add up to a smartphone to complete an augmented laboratory. ${ }^{36,37}$
Wang et al. reported an analytical device for the instantaneous semiquantitative detection of lead ions by combination of a smartphone and fluorescent paper strips. ${ }^{38}$ Nguyen et al. utilized a smartphone as a dark-field microscope for lead detection in drinking water. They mixed chromate ions with $\mathrm{Pb}^{2+}$ containing solutions to form lead chromate nanoparticles for colorimetric purposes. ${ }^{39}$ In previous studies of our group, a portable instrument for determination of mercury contamination in water samples was developed by measuring the $R G B$ color intensity delivered to the smartphone camera. $^{20}$

Herein, we developed a novel machine learning algorithm to upgrade the efficiency of smartphone-based colorimetry for lead ion detection and quantification. We designed a gadget and employed an extremely uniform intensity-light source with a specific $R G B$ pattern placed behind the reference and sample solutions. In the presence of $\mathrm{Pb}^{2+}$, the color of sample solution turns from red to purple in salty media because of aggregation of GNPs. The corresponding color changes between reference and sample solutions were captured by the smartphone camera. Then, an image processing procedure was used on the digital images for noise elimination and smoothing. Next, a feature extraction was performed on the corresponding $R G B$ values of experimental results to facilitate the subsequent machine learning procedure and model generalization.

In order to improve the accuracy and the efficiency of the lead concentration estimation, the current paper proposes an 

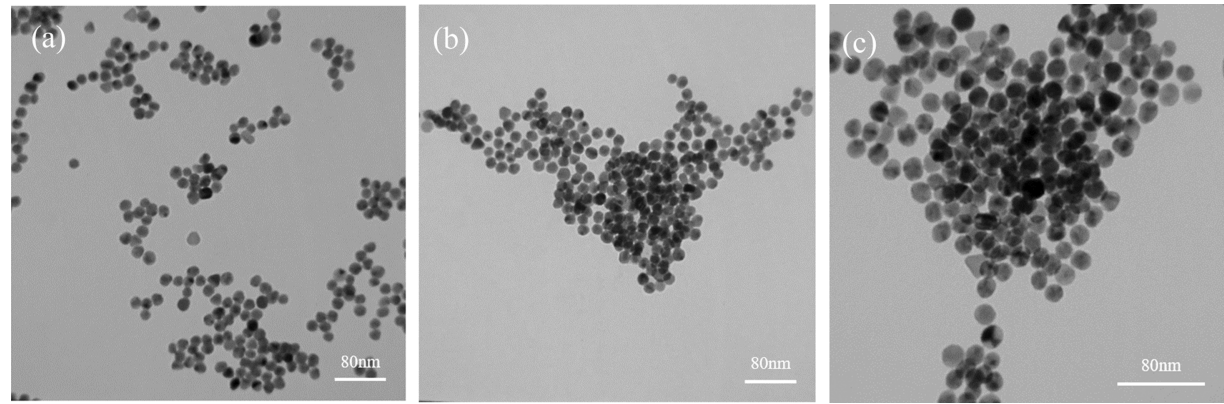

Figure 2. TEM images of aggregated GNPs at different concentrations of the $\mathrm{Pb}^{2+}$ in the sample solution: (a) 20, (b) 100, and (c) $200 \mathrm{ppb}$.
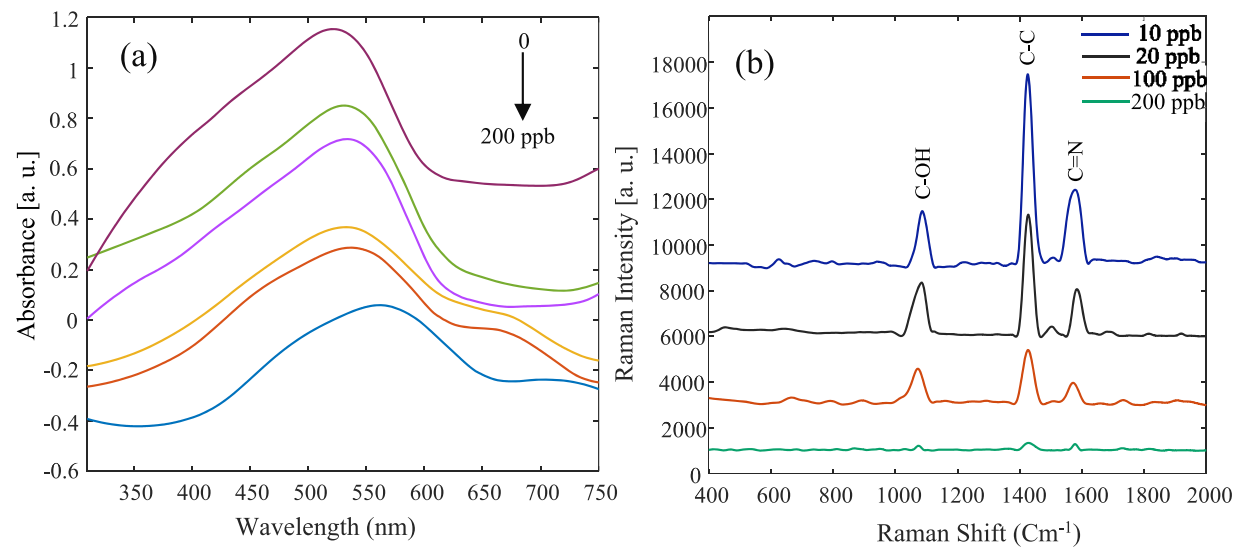

Figure 3. (a) UV-visible absorption spectra of the colorimetric system for detecting $\mathrm{Pb}^{2+}$ ions at various concentrations: blank, 10, 20, 50, 100, and $200 \mathrm{ppb}$ and (b) SERS spectra of the biosensor for different concentrations of $\mathrm{Pb}^{2+}$ ions.

approach based on the nonlinear regression method. Finally, the performance of the regression-based model was assessed based on the statistical values including average absolute error (AAE) and root mean square error (RMSE). The newly developed algorithm provides more than $40 \%$ improvement in the RMSE value, which indicates how close the experimental results are to the model's predicted values.

\section{RESULTS AND DISCUSSION}

Characterization of GNPs. The GNPs were synthesized by reducing $\mathrm{HAuCl}_{4}$ with sodium citrate. Then, $100 \mu \mathrm{L}$ volume of the GNP dispersion was pipetted onto a mesh grid (3 $\mathrm{mm}$ formvar/carbon coated grid) and dried at room temperature. The transmission electron microscopy (TEM) image, presented in Figure 1a reveals that the GNPs are spherical and monodisperse. The corresponding results from dynamic light scattering (DLS) measurements further demonstrated that all the obtained GNPs bear a uniformly spherical shape and with a narrow Gaussian size distribution. The average size was measured to be $18 \pm 1.2 \mathrm{~nm}$ (Figure $1 \mathrm{~b}$ ). Although larger-size nanoparticles lead to a more sensitive detection system, 15-20 nM GNPs were used because of their higher stability and reliability. In addition, LSPR peak shifts to a longer wavelength for larger-sized GNPs, and the sample solution's color turns to blue. Thus, the pre- and post-test color change is distinguishable hardly at larger-size GNP solution, which make them less desirable.

Visible absorption spectrums were obtained from 400 to 750 $\mathrm{nm}$ for the particles from the synthesis procedures and are shown in Figure 1c. Concentration of GNPs in a synthesized solution was estimated to be $9.7 \mathrm{nM}$ by UV-visible spectroscopy, and according to the Beer-Lambert law for 18 $\mathrm{nm}$ nanoparticles, the localized surface plasmon peak for GNPs appeared at around $525 \mathrm{~nm}$.

The X-ray diffraction (XRD) study of the dried solution confirmed the presence of GNPs with gold crystalline nature. The XRD pattern showed the presence of four characteristic intense peaks at $38.36,44.47,64.73$, and $77.65^{\circ}$, which correspond to (111), (200), (220), and (311) lattice planes (Figure 1d), respectively. The presence of intense peaks of nanoparticles confirms that the GNPs are crystalline in a natural way and suggest that the GNPs are stable and are not aggregated. ${ }^{40,41}$

Sensing Mechanisms for the Detection of $\mathrm{Pb}^{2+}$ lons. The guanine-rich oligonucleotide has the ability to adsorb spontaneously on the surface of GNPs and protect GNPs from aggregation in a salt saturated solution. In the presence of $\mathrm{Pb}^{2+}$ ions in an aqueous solution, the guanine-rich oligonucleotide would release into the solution to interact with $\mathrm{Pb}^{2+}$ and form a stable structure of the G-quadruplex. ${ }^{42}$ Thus, the bare GNPs remain unprotected and aggregate.

TEM images clarified that the morphology of the GNPs gradually changed from the spherical-shaped individual particles (Figure 1a) to the aggregated clusters with the increasing concentration of $\mathrm{Pb}^{2+}$ (Figure $2 \mathrm{a}-\mathrm{c}$ ).

UV-visible absorption spectra were obtained from 300 to $750 \mathrm{~nm}$ for the sample solutions. As shown in Figure 3a, when the concentration of $\mathrm{Pb}^{2+}$ was gradually increased, the maximum absorption peak of GNPs shifted from 525 to 575 $\mathrm{nm}$. Upon increasing $\mathrm{Pb}^{2+}$ concentration and GNP aggregation, the $525 \mathrm{~nm}$ peak broadens to form a new shoulder in the range of $670-750 \mathrm{~nm}$.

Figure $3 \mathrm{~b}$ provides the surface-enhanced Raman scattering (SERS) spectra of the proposed biosensor for different 
concentrations of $\mathrm{Pb}^{2+}$ ions. An obvious decrease in the intensity of characteristic peaks 1078,1428 , and $1587 \mathrm{~cm}^{-1}$ is assigned to the $\nu(\mathrm{C}-\mathrm{OH}), \nu(\mathrm{C}-\mathrm{C})$, and $\nu(\mathrm{C}=\mathrm{N})$ vibrations, respectively. ${ }^{42,43}$ Speciously, the $\mathrm{Pb}^{2+}$ ions interact with the oligonucleotides via their aforementioned functional groups. Thus, as the concentration of $\mathrm{Pb}^{2+}$ ions increased, the numbers of guanine-rich oligonucleotides on the GNP's surface decreased, and the Raman signal on the GNPs surface became weak.

Fourier transform infrared (FTIR) spectroscopy is also an important tool to investigate the presence of guanine-rich oligonucleotides on the GNPs' surface. FTIR spectra were measured in the absorbance mode by application of an average of 32 scans at a resolution of $4 \mathrm{~cm}^{-1}$ in the range of 500-4000 $\mathrm{cm}^{-1}$. The most common $\mathrm{KBr}$ pellet technique was used for recording solution spectra. For each sample, three drops were dripped onto one $\mathrm{KBr}$ pellet ( $25 \mathrm{~mm}$ diameter, $4 \mathrm{~mm}$ thick) and then sandwiched between the second $\mathrm{KBr}$ pellet. Having determined through our analysis of spacer thicknesses that good-quality spectra could be obtained at a path length of 10 $\mu \mathrm{M}$, next, we placed the pellets on the FTIR machine's sample holder for scanning. Absorbance values were baseline-corrected and smoothed. ${ }^{44}$

The FTIR spectra for the synthesized GNPs revealed two distinct peaks at 1632 and $3435 \mathrm{~cm}^{-1}$. The peak at 1632 is attributed to the $\mathrm{C}=\mathrm{O}$ group, and the second peak was identified to be a hydroxyl group, which indicates interaction with the water medium. ${ }^{45}$

In comparison, the peaks in the FTIR spectrum of functionalized GNPs in the presence of $\mathrm{Pb}^{2+}$ are shifted and weakened in intensities, which signifies that the guanine-rich oligonucleotides have the affinity to interact with GNPs, mainly through their oxygen functionalities which act as reducing and capping agents. The band at $2038 \mathrm{~cm}^{-1}$ indicated the presence of $\mathrm{C}-\mathrm{H}$ compounds on the nanoparticle surface. This means that the surface of GNPs is functionalized by guanine. The peak at $623 \mathrm{~cm}^{-1}$ was attributed to $\mathrm{C}-\mathrm{Br}$ stretching vibrations, and the peaks found at $1073 \mathrm{~cm}^{-1}$ could be assigned to $\mathrm{C}-\mathrm{O}$ stretching modes. ${ }^{46}$

As shown in Figure 4, obvious changes in the spectra before and after $\mathrm{Pb}^{2+}$ addition can be observed. The bands at 2038

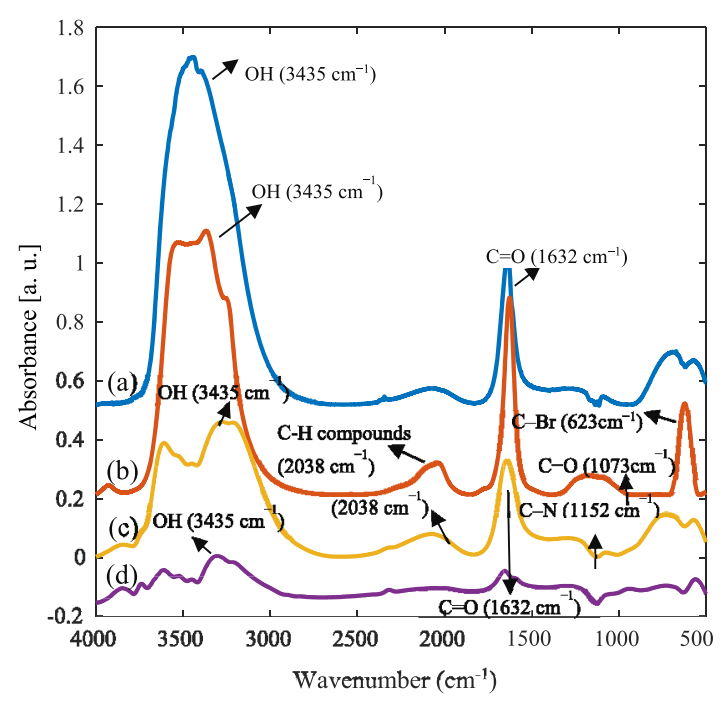

Figure 4. FTIR spectra of (a) GNPs, (b) functionalized GNPs, and sample solution with (c) 100 and (d) $200 \mathrm{ppb} \mathrm{Pb}^{2+}$ concentration. $\mathrm{cm}^{-1}$ strengthened. The intensity of the band of the $\mathrm{COOH}$ and $\mathrm{OH}$ groups at 3435 and $1663 \mathrm{~cm}^{-1}$ substantially decreased after $\mathrm{Pb}^{2+}$ binding. This result confirms that sulfur groups were involved in metal binding. The specific bonds in the functional groups that were found to shift include $\mathrm{O}-\mathrm{H}, \mathrm{C}-\mathrm{H}, \mathrm{C}=\mathrm{C}$, $\mathrm{C}=\mathrm{O}$, and $\mathrm{C}-\mathrm{O}$, which indicate that these specific functional groups involve in the adsorption of $\mathrm{Pb}^{2+}$ ions. ${ }^{46}$ Further, the changes in the vibrational frequencies around $1632 \mathrm{~cm}^{-1}$ show the attachment of metal ions with these functional groups. From the FTIR spectra, it is depicted that functional groups such as hydroxyl $(\mathrm{O}-\mathrm{H})$ and carbonyl groups $(\mathrm{C}=\mathrm{O})$ were responsible for adsorption of metal ions as changes in the peaks were observed around the wavelengths of these groups. The absorbance bands at $1152 \mathrm{~cm}^{-1}$ corresponded to the stretching of the weakened $\mathrm{C}-\mathrm{N}$ bond and shifted to a higher wave number, which indicates the formation of the $\mathrm{N}-\mathrm{Pb}^{2+}$ bond in the adsorption process.

Concentration Estimation Via Machine Learning. A model that estimates the accurate concentration of $\mathrm{Pb}^{2+}$ ions in aqueous solutions was recently developed by analyzing the smartphone-captured images $(3096 \times 4128$ pixels $)$. By increasing the concentration of $\mathrm{Pb}^{2+}$ ions, more agglomeration occurs in the solution and as a result, the GNPs interact with larger wavelengths. Thus, the absorption of red color in the solution is increased, and lower intensity is delivered to the smartphone camera. As illustrated in Figure 5a, the blue- and green-color lights experience an opposite situation. Their interaction with larger wavelengths decreases upon $\mathrm{Pb}^{2+}$ increment, which in turn delivers more intense colors to the smartphone camera.

The newly extracted features are created with respect to the properties of the original data and ultimately improve predictive accuracy of the model. The modified version of the rectifier linear unit (ReLU) nonlinear function is used to perform nonlinear transformations on the desired data set over a given interval. The values in the range of $[0,1]$ were chosen for feature extraction with respect to $R G B$ channels' data. Each transformation tries to robust the effect of some features, their numerical value of which is close to the original data. The features which are far away from data values become zero. Figure $5 \mathrm{~b}$ shows the extracted features of the reconstructed images.

A regression-based model has been proposed for the first time to estimate the $\mathrm{Pb}^{2+}$ concentration in aqueous medium. This newly designed machine's regression parameters were learned by optimizing RMSE loss. Equation 1 predicts the concentration as a function of $R(r, g, b), G(r, g, b)$, and $B(r, g, b)$.

$$
\operatorname{Ln}\left(\mathrm{Pb}^{2+} \text { concentration }\right)=\beta_{i} X_{i, j}^{\prime \prime}
$$

$\beta_{i}$ is the $1 \times 18$ vector of nonlinear regression coefficients. A plot of regression coefficients is illustrated in Figure 6. The concentration of $\mathrm{Pb}^{2+}$ can be predicted by inserting the coefficient values into eq 1 .

Prior to the regression analysis, the database was subdivided into two parts containing 80 and $20 \%$ for the training and test data sets, respectively. This split up was obtained in a random fashion for systematic evaluation of the proposed technique. Figure 7 shows the predicted values of training and testing sets versus experimental data and demonstrates a good agreement between experimental and predicted data.

The analytical performance of the regression-based model was compared with previous works (Table 1). ${ }^{20,21}$ The 

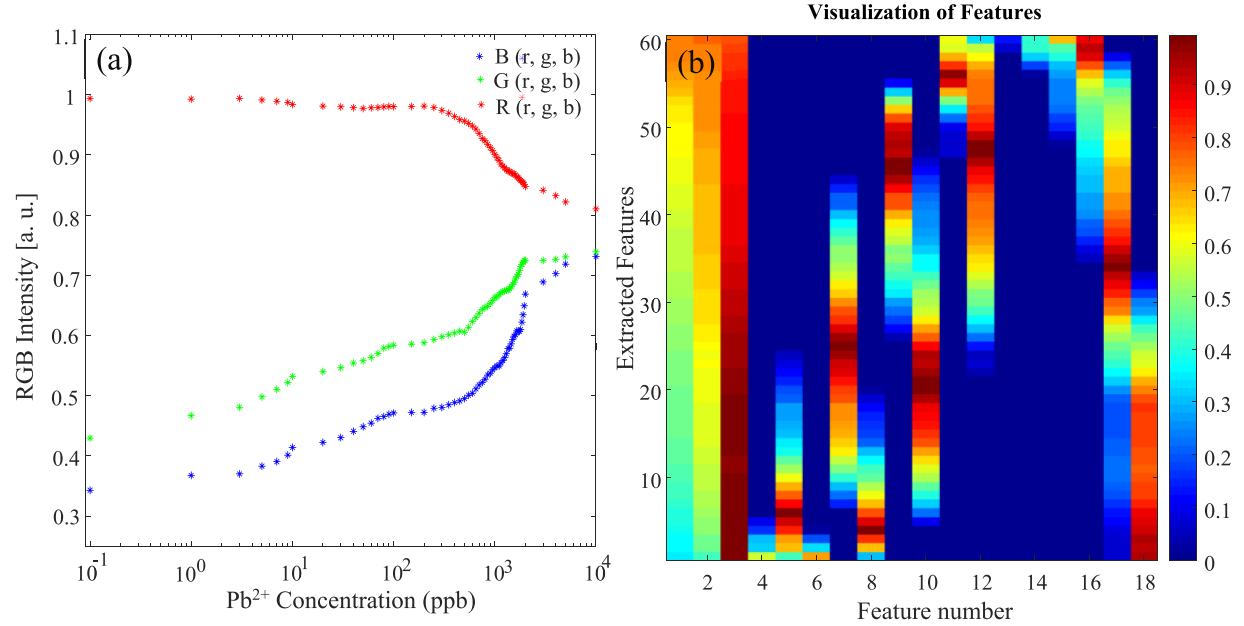

Figure 5. (a) $R G B$ intensity of the input data matrix $\left(X_{i, j}\right)$ and (b) extracted features of images.

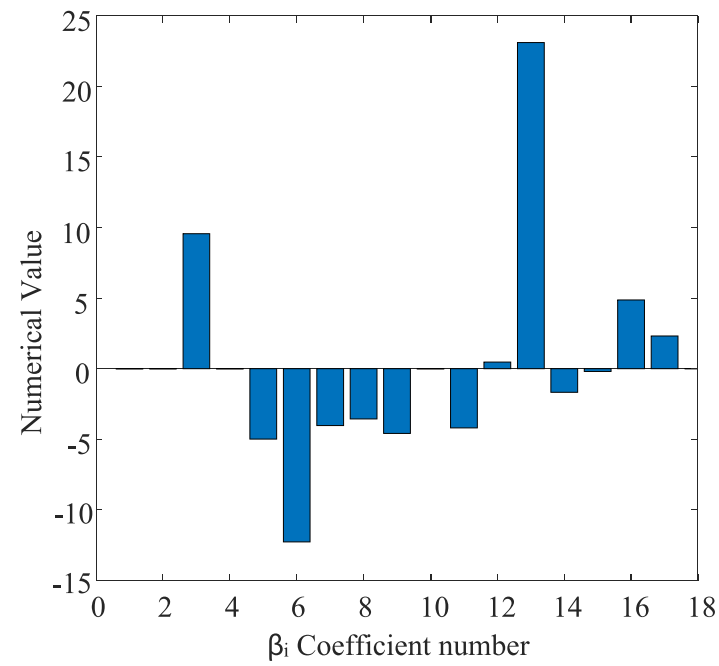

Figure 6. Representation of the regression coefficients of eq 1 for $\mathrm{Pb}^{2+}$ concentration estimation.

practical limit of detection was improved by nearly 8 orders of magnitude in comparison to the ratiometric method using the nonlinear regression method.
Table 1. Comparison between the Analytical Characteristic of the Proposed Machine Learning Based Approach and the Ratiometric Method for $\mathbf{P b}^{2+}$ Detection

\begin{tabular}{|c|c|c|c|}
\hline \multirow[b]{2}{*}{ figure of merit } & \multirow[b]{2}{*}{$\begin{array}{l}\text { ratiometric } \\
\text { method }^{21}\end{array}$} & \multicolumn{2}{|c|}{ machine learning-based method } \\
\hline & & $\begin{array}{l}\text { linear } \\
\text { regression }\end{array}$ & $\begin{array}{l}\text { nonlinear } \\
\text { regression }\end{array}$ \\
\hline linear range & $2-20 \mathrm{ppb}$ & $3.5-1000 \mathrm{ppb}$ & $0.5-2000 \mathrm{ppb}$ \\
\hline $\operatorname{RSD}(n=3)$ & $\leq 2.7 \%$ & $\leq 2.3 \%$ & $\leq 2.1 \%$ \\
\hline $\begin{array}{l}\text { limit of } \\
\text { detection }\end{array}$ & $4 \mathrm{ppb}$ & $2.7 \mathrm{ppb}$ & $0.5 \mathrm{ppb}$ \\
\hline RMSE & & 0.1754 & 0.1244 \\
\hline $\mathrm{AAE}$ & & 0.1467 & 0.0943 \\
\hline
\end{tabular}

Also, the enhancement in statistical values of $\mathrm{AAE}$ and RMSE indicates how close the experimental results are to the model's predicted values. The linear range of sensor ranged from 0.5 to $2000 \mathrm{ppb}$ for the predicted data sets, which illustrates the new model can precisely predict the $\mathrm{Pb}^{2+}$ concentration in a wide range.

Optimization of Sensing Conditions. The effect of different experimental conditions such as temperature, $\mathrm{pH}$, oligonucleotide concentration, and reaction time were analyzed and optimized to have the best response. Variation
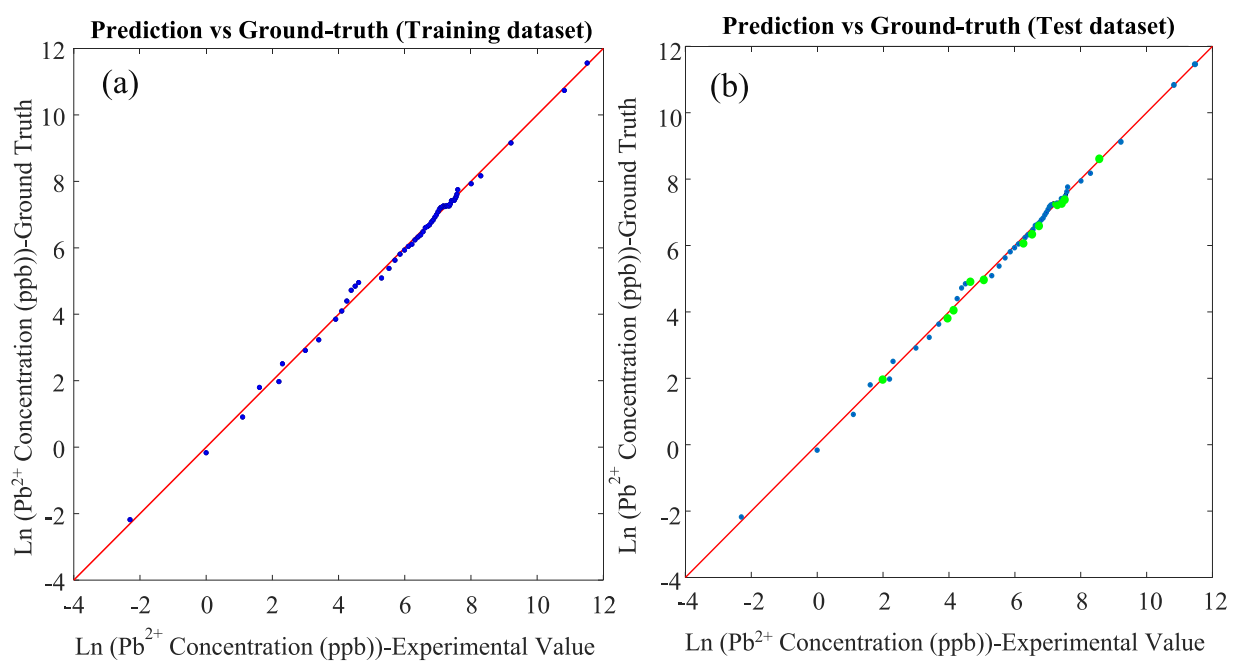

Figure 7. (a) Training data and (b) testing data for $\mathrm{Pb}^{2+}$ concentration estimation (ppb). 

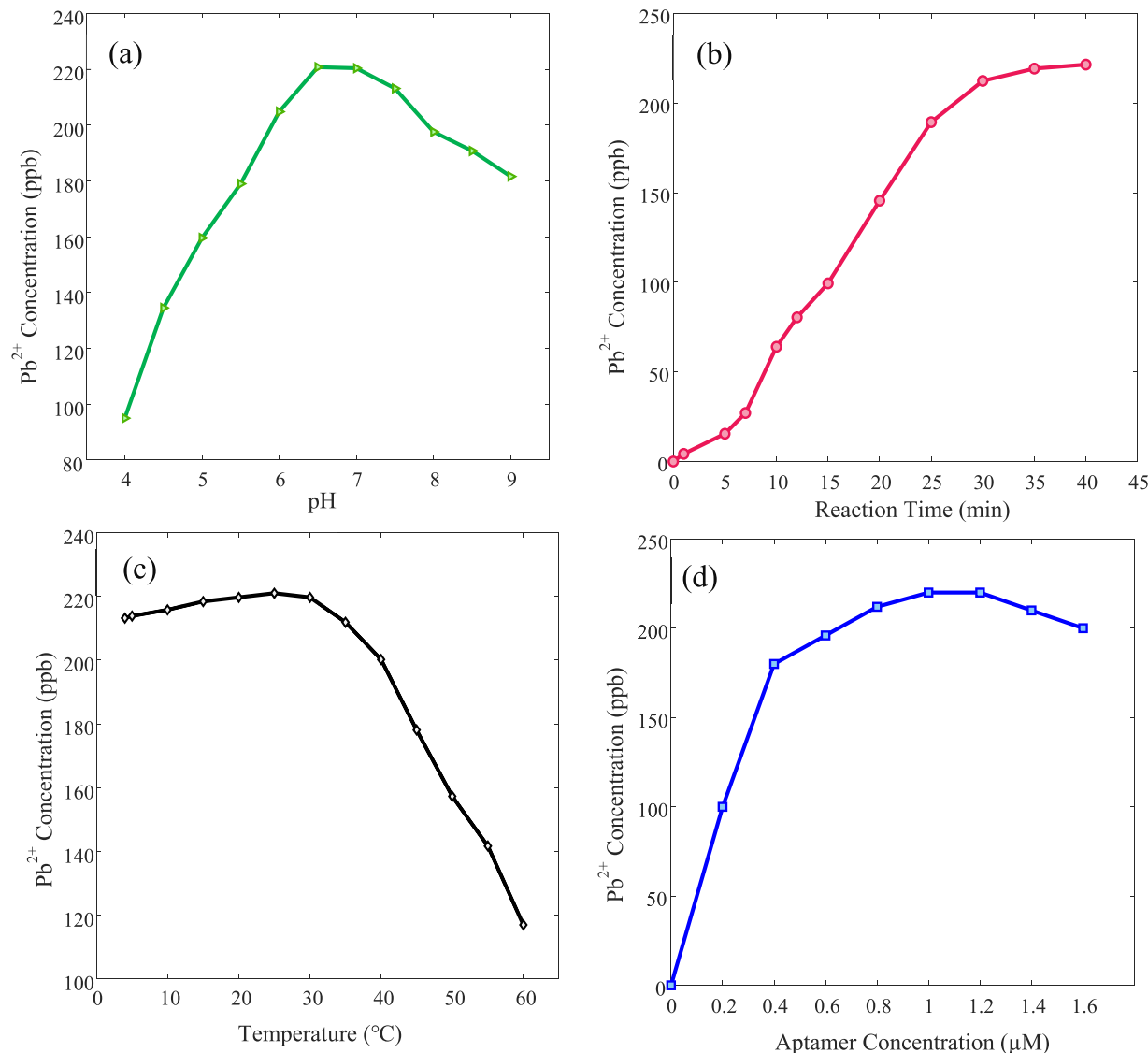

Figure 8. Optimization of the experimental parameters: effects of (a) $\mathrm{pH}$, (b) reaction time, (c) temperature, and (d) oligonucleotide concentration on the sensor response.

Table 2. Performance of the Smartphone-Based Method for $\mathrm{Pb}^{2+}$ Detection in Real Samples ${ }^{a}$

\begin{tabular}{|c|c|c|c|c|c|c|c|}
\hline \multirow[b]{2}{*}{ real sample } & \multirow[b]{2}{*}{ added (ppb) } & \multicolumn{3}{|c|}{ ICP-MS method } & \multicolumn{3}{|c|}{ smartphone-based method } \\
\hline & & found $(\mathrm{ppb})$ & recovery $(\%)$ & $\operatorname{RSD}(n=3)$ & found $(\mathrm{ppb})$ & recovery (\%) & $\operatorname{RSD}(n=3)$ \\
\hline \multirow[t]{3}{*}{ tap water } & 0.15 & & & & 0.13 & 86 & 2.7 \\
\hline & 1.5 & 1.7 & 113 & 2.6 & 1.5 & 100 & 1.6 \\
\hline & 15 & 16.2 & 108 & 3 & 15.8 & 105 & 1.9 \\
\hline \multirow[t]{3}{*}{ river water } & 0.15 & & & & 0.16 & 106 & 2.1 \\
\hline & 1.5 & 1.4 & 93 & 2.1 & 1.4 & 93 & 1.5 \\
\hline & 15 & 15.2 & 100 & 1.5 & 15.2 & 101 & 1.2 \\
\hline \multirow[t]{3}{*}{ Zamzam water } & 0.15 & & & & 0.11 & 73 & 3.2 \\
\hline & 1.5 & 1.6 & 106 & 2.8 & 1.3 & 87 & 1.9 \\
\hline & 15 & 15.2 & 101 & 3.1 & 14 & 93 & 2.4 \\
\hline
\end{tabular}

${ }^{a}$ Each test was repeated three times, and the average of three repeat \pm standard deviation is reported.

of the water samples' $\mathrm{pH}$ between 4 and 9 via the addition of sodium hydroxide $2 \mathrm{M}$ and acetic acid $5 \mathrm{M}$ to the dispersions while fixing the $\mathrm{Pb}^{2+}$ concentration at $220 \mathrm{ppb}$ was investigated. Figure 8a illustrates how $\mathrm{pH}$ of the water sample affects the sensor response. This behavior can be explained by the protonation of guanine groups of the aptamer sequence while $\mathrm{pH}<7.0$ and the increase in negative charge on aptamer while $\mathrm{pH}>8.0$, which leads to disassembly of G-quartets of the aptamer. $^{47}$

The changes between the reference and sample solution were recorded by the smartphone camera during $40 \mathrm{~min}$. At room temperature, the response appears after $3 \mathrm{~min}$ and then gradually increases with increasing reaction time. According to Figure $8 b$, the response increases with the increasing reaction time and then remains constant after $30 \mathrm{~min}$. Thus, $30 \mathrm{~min}$ is the minimum required time for this analysis.

Next, another study was performed to investigate the temperature dependence of responses between 4 and $60{ }^{\circ} \mathrm{C}$. As shown in Figure $8 \mathrm{c}$, the response decreases above $40{ }^{\circ} \mathrm{C}$ suddenly which is because of the fact that the aptamer cannot maintain its folded structure when the temperature rises above the melting point, and G-quarter structure is more stable at low temperatures. ${ }^{47,48}$ The response therefore is stable from 20 to $35{ }^{\circ} \mathrm{C}$.

The concentration of oligonucleotides in the range of $0.1-$ $1.6 \mu \mathrm{M}$ was investigated to check how it affects the detection of $\mathrm{Pb}^{2+}$ ions. Figure $8 \mathrm{~d}$ shows that low concentration of oligonucleotides is not enough for binding to the $\mathrm{Pb}^{2+}$ ions. In contrast, at higher concentrations, the excessive oligonucleo- 
tides acted as electrostatic barriers. ${ }^{49}$ The optimal concentration of $\mathrm{NaCl}$ was chosen to be $100 \mathrm{mM}$ (Figure S1).

Real Sample Analysis. To validate the capability of the proposed method for real sample analysis, samples were taken from different sources, including: tap water (Tehran, Iran), river water (Mazandaran, Iran), and Zamzam water (Mecca, Saudi Arabia). Initially, the samples without dilution were investigated, and then, samples were spiked with $\mathrm{Pb}^{2+}$ ion solutions in order to make $0.15,1.5$, and $15 \mathrm{ppb}$ solutions. The analytical results, which are presented in Table 2 , demonstrate the average recovery percent of the spiked samples was better than $93.7 \%$. To compare the accuracy of smartphone-based results with a common method, ICP-MS analysis has been performed to identify the $\mathrm{Pb}^{2+}$ concentration in the spiked samples. Results of the smartphone-based method showed a remarkable consistency with those obtained by ICP-MS measurements.

Interference Effect. The selectivity of the analytical method was examined in the presence of various interfering ions. The concentration of all metal ions was prepared to be $220 \mathrm{ppb}$. As shown in Figure 9, the analysis of results

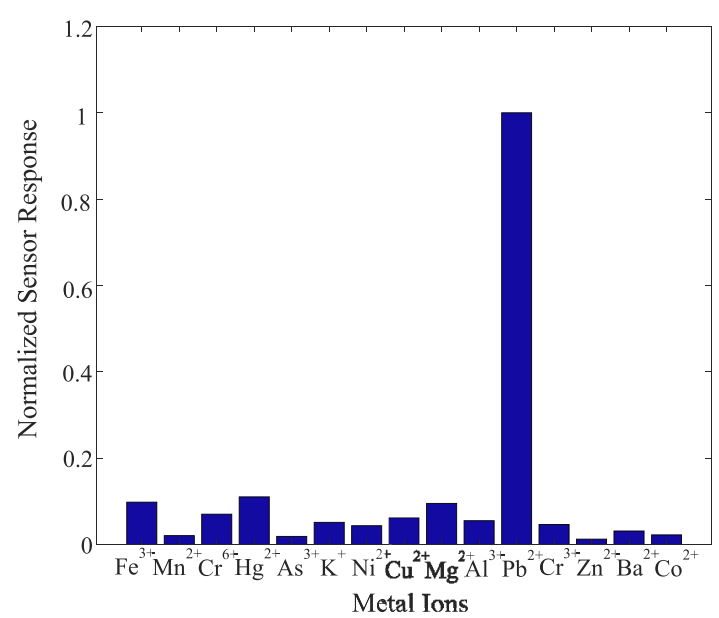

Figure 9. Specificity of the sensor toward detection of $\mathrm{Pb}^{2+}$ and interfering ions at the concentration of $220 \mathrm{ppb}$.

demonstrates that the $\mathrm{Pb}^{2+}$ sensor response is negligible for other added metal ions, and only the sample containing $\mathrm{Pb}^{2+}$ produces a remarkable change. Observation illustrates crossreactivity was less than $10 \%$ in the presence of additional ions, and it provides an influential implement for real sample tests.

\section{CONCLUSIONS}

In this work, a new method for detection of $\mathrm{Pb}^{2+}$ ions in the aqueous solutions is proposed by using processed $R G B$ images and machine learning approach on a mobile platform. The color changes from red wine to purple as a result of GNP aggregation captured by a smartphone camera. A model was created and learned to explain $\mathrm{Pb}^{2+}$ concentration as a function of $R(r, g, b), G(r, g, b)$, and $B(r, g, b)$ parameters in the captured images. The regression model was trained through machine learning on $R G B$ values and extracted features. The nonlinear regression was applied, and the concentration of $\mathrm{Pb}^{2+}$ was successfully estimated. The statistical values between machine learning-predicted and experimental results are 0.1244 and 0.0943 for RMSE and AAE, respectively.
Under optimal conditions, the colorimetric method has high sensitivity for $\mathrm{Pb}^{2+}(\mathrm{LOD}=0.5 \mathrm{ppb})$ with short detection time. In order to obtain the best sensor response, the oligonucleotide binding conditions and as oligonucleotide concentrations were optimized. Selectivity observations indicated cross-reactivity was less than $10 \%$ in the presence of various interfering ions. The biosensor's results were also verified by the well-known ICP-MS approach. As a result, the proposed method provides an influential tool for real-life samples.

\section{EXPERIMENTAL SECTION}

Reagents and Apparatus. Hydrogen tetrachloroaurate $\left(\mathrm{HAuCl}_{4} \cdot 3 \mathrm{H}_{2} \mathrm{O}\right)$, trisodium citrate $\left(\mathrm{Na}_{3} \mathrm{C}_{6} \mathrm{H}_{5} \mathrm{O}_{7}\right)$, sodium chloride $(\mathrm{NaCl})$, and lead(II) nitrate $\left(\mathrm{Pb}\left(\mathrm{NO}_{3}\right)_{2}\right)$ were purchased from Sigma-Aldrich. Single oligonucleotides ( $5^{\prime}$ GGTTGGGCGGGATGGGTG-3') have been used as the aptamer from Roobin Teb Gostar. The stock solution of $\mathrm{Pb}^{2+}$ $(1 \mathrm{mM})$ was prepared by dissolving $\mathrm{Pb}\left(\mathrm{NO}_{3}\right)_{2}$ in ultrapure $(18.2 \mathrm{M} \Omega \cdot \mathrm{cm})$ water (Milli-Q, Millipore).

The TEM images were obtained from Philips CM30, operating at an accelerating voltage of $200 \mathrm{kV}$. DLS measurements of the particle size distribution were performed using Horiba SZ-100. The absorption spectra were obtained at room temperature by a Photonix Ar 2015 UV-visible spectrometer in a quartz cell with a path of $1.0 \mathrm{~cm}$.

FTIR spectra before and after $\mathrm{Pb}^{2+}$ addition were recorded on a PerkinElmer spectrometer, Spectrum Two. XRD pattern was recorded using X'Pert Pro equipped with a $\mathrm{Cu} \mathrm{K} \alpha$ source of $1.5405 \AA$ wavelength. ICP-MS measurements were carried out on PerkinElmer Elan 6100 inductively coupled mass spectrometer. SERS spectra were collected using a Horiba Xplora plus Raman spectroscope using $785 \mathrm{~nm}$ laser for excitation.

A digital pH meter (Metrohm $827 \mathrm{pH} \mathrm{lab)} \mathrm{was} \mathrm{employed}$ for $\mathrm{pH}$ measurements. The light source was a full color TFT LCD, which has a display of 2.4 inch with $320 \times 240$ pixel resolution. The digital images for the colorimetric analysis was recorded by a Samsung Galaxy A3, which has a 13 megapixel back camera with an aperture size of $f / 1.9$. Because the higher ISO speeds dramatically increase the image noise, the ISO parameter was set to 100 for all images (Table S1).

Sample Preparation. The colloidal GNPs were synthesized via a two-step Turkevich reduction process. ${ }^{50}$ In this method, the hydrogen tetrachloroaurate solution was used as the $\mathrm{Au}^{3+}$ ion precursor, which is reduced to $\mathrm{Au}^{0}$ in the presence of sodium citrate as a reducing and stabilizing agent. The overall reaction time could be determined by observing the solution until a characteristic red wine color was obtained. ${ }^{50}$

The thiolated oligonucleotide was used for conjugation with GNPs. First, $30 \mu \mathrm{L}$ of $1 \mu \mathrm{M}$ oligonucleotides was added to 120 $\mu \mathrm{L}$ of GNP solution $(9.7 \mathrm{nM})$. The prepared solution was allowed to react for $20 \mathrm{~min}$ at room temperature. Next, $100 \mu \mathrm{L}$ of $\mathrm{Pb}^{2+}$ solution was added and mixed for $30 \mathrm{~min}$. A total of 60 solutions with different $\mathrm{Pb}^{2+}$ concentrations have been used to construct the calibration curve. The concentrations ranging from $0.1 \mathrm{ppb}$ to $10 \mathrm{ppm}$ of $\mathrm{Pb}^{2+}$ solutions were prepared by using serial dilution of the stock solution. Finally, $70 \mu \mathrm{L}$ of 100 $\mathrm{mM} \mathrm{NaCl}$ solution was added.

Smartphone-Based Colorimetric Reading System. A light-weight attachment housing was used based on the previous design. $^{20}$ It incorporates as the holder and basic optical element for colorimetric purposes. The schematic of the final design of the device is shown in Figure 10. The 3D 
(a)

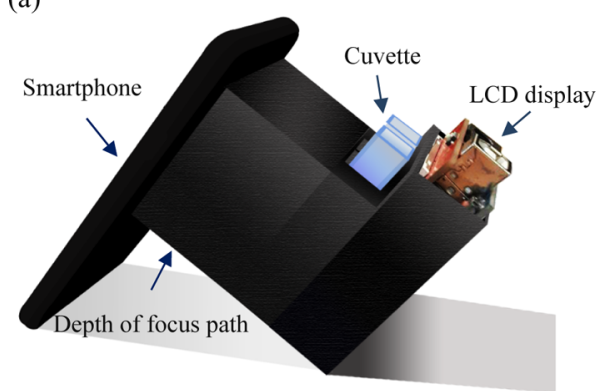

(b)

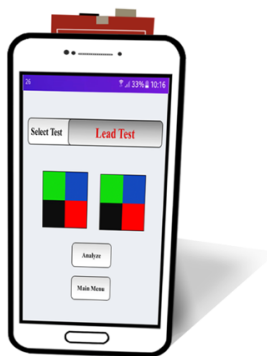

Figure 10. (a) Schematic of the designed smartphone-based colorimetry tool and (b) custom-developed smartphone application for real-time and on-site monitoring of $\mathrm{Pb}^{2+}$ content.

printing material was opaque black polylactic acid in order to block ambient light interference and thus provided a reproducible LCD illumination. A full-color TFT LCD provides an ultrastable and uniform intensity to serve as an excitation source. An Arduino UNO microcontroller board drives the TFT LCD and makes it possible to display any desired pattern. Some examples of digital images captured by the smartphone are presented in Supporting Information Figure S2.

Feature Extraction and Machine Learning. The concentration of $\mathrm{Pb}^{2+}$ can be estimated precisely using our new machine learning algorithm and extracted features. Three variables (the mean value of red, green, and blue readings) were considered as the inputs, and the $\mathrm{Pb}^{2+}$ concentration was regressed using nonlinear regression.

In the input data matrix $\left(X_{i, j}\right)$ given in eq 2 , the rows refer to the number of samples $1 \leq i \leq 60$, and the columns show $R G B$ color channels $1 \leq j \leq 3$. To obtain, for example, $r_{1}$, we placed a $200 \times 200$-pixel square in the red part of the sample cuvette image. Then, we calculated the variation of the average red intensities between reference and sample solutions. Similar calculations were carried out for $g_{1}$ and $b_{1}$ in the green and the blue areas of the image, respectively.

$$
X_{i, j}=\left[\begin{array}{lll}
r_{1} & g_{1} & b_{1} \\
r_{2} & g_{2} & b_{2} \\
\vdots & \vdots & \vdots \\
r_{60} & g_{60} & b_{60}
\end{array}\right]
$$

Two-dimensional convolution operation between $\mathrm{X}_{i, j}$ and $\mathrm{a}$ filter $(B)$ was performed for smoothing and noise elimination. The filter was applied pixel by pixel using the kernel described by the eq 3. Actually, if the value of a pixel in the digital images is far from its neighbors' average pixels, the designed filter detects and removes it. The convolution filters use local neighbors to compute the weighted average, and each pixel is used multiple times by its neighbors. The following equation defines the convolution of $\mathrm{X}_{i, j}$ and $B$

$$
\begin{gathered}
X_{i, j}^{\prime}=B \times X_{i, j}=\sum_{s=-a}^{a} \sum_{t=-b}^{b} B(s, t) X(i-s, j-t) \\
B^{T}=[1 / 5, \ldots, 1 / 5]^{T},\left|B^{T}=5\right|
\end{gathered}
$$

$X_{i, j}^{\prime}$ is the filtered data matrix.

Most of the smartphone-based colorimetric methods rely on $R G B$ data extracted from captured images, and the efforts are mainly devoted to correct interpretation of results. Feature extraction is the basis for high-performance detection using machine learning methods because the detection models have to be optimized. Feature extraction is the process of using domain knowledge about the data in order to extract potentially useful features. ${ }^{51,52}$

Herein, a new feature extraction method was used to achieve high-performance learning algorithms that ultimately improve predictive accuracy of the model. The ReLU, which is an element-wise nonlinear function, was used for feature extraction. ${ }^{53}$ ReLU function gives $x$ to the output if $x$ is positive and 0 otherwise. The modified version of the famous nonlinear function ReLU is used to extract new features, which is presented in eq 4

$$
X_{i, j}^{\prime \prime}(i)=\max \left(1-\frac{\left|X_{i, j}^{\prime}-i\right|}{0.1}, 0\right) \quad i \in[0: 0.1: 1]
$$

Applying this method leads to the addition of new data sets of features to the experimental results stored in the $X_{i, j}^{\prime \prime}$ matrix. $\mathrm{Pb}^{2+}$ concentrations (target values) are stored in the $Y$ matrix as follows

$$
Y=\left[\begin{array}{l}
0.1 \\
1 \\
\vdots \\
100000
\end{array}\right]
$$

Nonlinear regression is widely applied to examine observed relations among pairs of variables and to compare these with the theoretical relations. The goal is to find the functional relationship between the inputs ( $R G B$ values) and output $\left(\mathrm{Pb}^{2+}\right.$ concentration $)$. The regression model is as follows

$$
Y_{\text {prd }}=\beta_{i} X_{i, j}^{\prime \prime} \quad i=0,1, \ldots, k
$$

where $Y_{\mathrm{prd}}$ is the predicted value of concentration, and $\beta_{i}$ is the unknown nonlinear regression coefficient to be calculated. The performance of the regression-based model is assessed based on the statistical values, AAE, and RMSE. ${ }^{54}$ The equations to calculate these statistical values are

$$
\mathrm{AAE}=\frac{1}{N} \sum_{i=1}^{N}\left|Y_{\mathrm{prd}, i}-Y_{\mathrm{exp}, i}\right|
$$

In order to estimate the coefficients $\left(\beta_{i}\right)$, the RMSE loss function was optimized. 


$$
\text { RMSE }=\sqrt{\frac{1}{N} \sum_{i=1}^{N}\left(\left|Y_{\text {prd }, i}-Y_{\text {exp }, i}\right|\right)^{2}}
$$

where $Y_{\text {exp, } i}$ is the experimental value, and $N$ is the number of data points in the data set.

\section{ASSOCIATED CONTENT}

\section{(s) Supporting Information}

The Supporting Information is available free of charge at https://pubs.acs.org/doi/10.1021/acsomega.0c04255.

Characteristics of the smartphone's main camera; $\mathrm{NaCl}$ effect on the biosensor's performance; digital images; and $R G B$ values $(\mathrm{PDF})$

\section{AUTHOR INFORMATION}

\section{Corresponding Author}

Mohammadreza Kolahdouz - School of Electrical and Computer Engineering, College of Engineering, University of Tehran, Tehran, Iran; ๑ orcid.org/0000-0001-6992-6950; Email: Kolahdouz@ut.ac.ir

\section{Authors}

Samira Sajed - School of Electrical and Computer Engineering, College of Engineering, University of Tehran, Tehran, Iran

Mohammad Amin Sadeghi - School of Electrical and Computer Engineering, College of Engineering, University of Tehran, Tehran, Iran

Seyedeh Fatemeh Razavi - School of Electrical and Computer Engineering, College of Engineering, University of Tehran, Tehran, Iran

Complete contact information is available at:

https://pubs.acs.org/10.1021/acsomega.0c04255

\section{Notes}

The authors declare no competing financial interest.

\section{ACKNOWLEDGMENTS}

The authors would like to thank the University of Tehran Science and Technology Park for the financial support (grant no. 9858019$)$.

\section{REFERENCES}

(1) Li, X.; Zhang, X.; Wang, X.; Yang, X.; Cui, Z. BioaugmentationAssisted Phytoremediation of Lead and Salinity Co-Contaminated Soil by Suaeda Salsa and Trichoderma Asperellum. Chemosphere 2019, 224, 716-725.

(2) Mazumdar, M.; Bellinger, D. C.; Gregas, M.; Abanilla, K.; Bacic, J.; Needleman, H. L. Low-Level Environmental Lead Exposure in Childhood and Adult Intellectual Function: A Follow-up Study. Environ. Health 2011, 10, 24.

(3) Zhang, Y.; Leng, Y.; Miao, L.; Xin, J.; Wu, A. The colorimetric detection of $\mathrm{Pb} 2+$ by using sodium thiosulfate and hexadecyl trimethyl ammonium bromide modified gold nanoparticles. Dalton Trans. 2013, 42, 5485-5490.

(4) Barnett, N. W.; Chen, L. S.; Kirkbright, G. F. Determination of Trace Concentrations of Lead and Nickel in Freeze-Dried Human Milk by Atomic Absorption Spectrometry and Inductively-Coupled Plasma Emission Spectrometry. Anal. Chim. Acta 1983, 149, 115121.

(5) de Godoi Pereira, M.; Arruda, M. A. 1. Z. Preconcentration of $\mathrm{Cd}(\mathrm{II})$ and $\mathrm{Pb}(\mathrm{II})$ Using Humic Substances and Flow Systems Coupled to Flame Atomic Absorption Spectrometry. Microchim. Acta 2004, 146, 215-222.
(6) Zhang, D.; Yang, S.; Ma, Q.; Sun, J.; Cheng, H.; Wang, Y.; Liu, J. Simultaneous Multi-Elemental Speciation of $\mathrm{As}, \mathrm{Hg}$ and $\mathrm{Pb}$ by Inductively Coupled Plasma Mass Spectrometry Interfaced with HighPerformance Liquid Chromatography. Food Chem. 2020, 313, 126119.

(7) Ebdon, L.; Hill, S. J.; Rivas, C. Lead Speciation in Rainwater by Isotope Dilution-High Performance Liquid Chromatography-Inductively Coupled Plasma-Mass Spectrometry. Spectrochim. Acta, Part B 1998, 53, 289-297.

(8) Byers, H. L.; McHenry, L. J.; Grundl, T. J. XRF Techniques to Quantify Heavy Metals in Vegetables at Low Detection Limits. Food Chem. X 2019, 1, 100001.

(9) Al Maliki, A.; Al-lami, A. K.; Hussain, H. M.; Al-Ansari, N. Comparison between Inductively Coupled Plasma and X-Ray Fluorescence Performance for $\mathrm{Pb}$ Analysis in Environmental Soil Samples. Environ. Earth Sci. 2017, 76, 433.

(10) Beqa, L.; Singh, A. K.; Khan, S. A.; Senapati, D.; Arumugam, S. R.; Ray, P. C. Gold Nanoparticle-Based Simple Colorimetric and Ultrasensitive Dynamic Light Scattering Assay for the Selective Detection of $\mathrm{Pb}(\mathrm{II})$ from Paints, Plastics, and Water Samples. ACS Appl. Mater. Interfaces 2011, 3, 668-673.

(11) Lin, Z.; Chen, Y.; Li, X.; Fang, W. $\mathrm{Pb}^{2+}$ Induced DNA Conformational Switch from Hairpin to G-Quadruplex: Electrochemical Detection of $\mathrm{Pb}^{2+}$. Analyst 2011, 136, 2367-2372.

(12) Li, B. L.; Wang, J.; Zou, H. L.; Garaj, S.; Lim, C. T.; Xie, J.; Li, N. B.; Leong, D. T. Low-Dimensional Transition Metal Dichalcogenide Nanostructures Based Sensors. Adv. Funct. Mater. 2016, 26, 7034-7056.

(13) Liu, Z.; Jin, W.; Wang, F.; Li, T.; Nie, J.; Xiao, W.; Zhang, Q.; Zhang, Y. Ratiometric Fluorescent Sensing of $\mathrm{Pb}^{2+}$ and $\mathrm{Hg}^{2+}$ with Two Types of Carbon Dot Nanohybrids Synthesized from the Same Biomass. Sens. Actuators, B 2019, 296, 126698.

(14) Qi, Y.; Ma, J.; Chen, X.; Xiu, F.-R.; Chen, Y.; Lu, Y. Practical Aptamer-Based Assay of Heavy Metal Mercury Ion in Contaminated Environmental Samples: Convenience and Sensitivity. Anal. Bioanal. Chem. 2020, 412, 439-448.

(15) Son, H.; Lee, H. Y.; Lim, J. M.; Kang, D.; Han, W. S.; Lee, S. S.; Jung, J. H. A Highly Sensitive and Selective Turn-On Fluorogenic and Chromogenic Sensor Based on BODIPY-Functionalized Magnetic Nanoparticles for Detecting Lead in Living Cells. Chem.-Eur. J. 2010, 16, 11549-11553.

(16) Priyadarshini, E.; Pradhan, N. Gold Nanoparticles as Efficient Sensors in Colorimetric Detection of Toxic Metal Ions: A Review. Sens. Actuators, B 2017, 238, 888-902.

(17) Anwar, A.; Minhaz, A.; Khan, N. A.; Kalantari, K.; Afifi, A. B. M.; Shah, M. R. Synthesis of gold nanoparticles stabilized by a pyrazinium thioacetate ligand: A new colorimetric nanosensor for detection of heavy metal Pd(II). Sens. Actuators, B 2018, 257, 875881 .

(18) Maier, S. A. Plasmonics: Fundamentals and Applications; Springer Science \& Business Media, 2007.

(19) Godakhindi, V. S.; Kang, P.; Serre, M.; Revuru, N. A.; Zou, J. M.; Roner, M. R.; Levitz, R.; Kahn, J. S.; Randrianalisoa, J.; Qin, Z Tuning the Gold Nanoparticle Colorimetric Assay by Nanoparticle Size, Concentration, and Size Combinations for Oligonucleotide Detection. ACS Sens. 2017, 2, 1627-1636.

(20) Sajed, S.; Arefi, F.; Kolahdouz, M.; Sadeghi, M. A. Improving Sensitivity of Mercury Detection Using Learning Based Smartphone Colorimetry. Sens. Actuators, B 2019, 298, 126942.

(21) Xu, H.; Liu, B.; Chen, Y. A colorimetric method for the determination of lead(II) ions using gold nanoparticles and a guaninerich oligonucleotide. Microchim. Acta 2012, 177, 89-94.

(22) Liu, J.; Lu, Y. A Colorimetric Lead Biosensor Using DNAzymeDirected Assembly of Gold Nanoparticles. J. Am. Chem. Soc. 2003, $125,6642-6643$

(23) Slocik, J. M.; Zabinski, J. S., Jr; Phillips, D. M.; Naik, R. R. Colorimetric Response of Peptide-Functionalized Gold Nanoparticles to Metal Ions. Small 2008, 4, 548-551. 
(24) Chai, F.; Wang, C.; Wang, T.; Li, L.; Su, Z. Colorimetric Detection of $\mathrm{Pb}^{2+}$ Using Glutathione Functionalized Gold Nanoparticles. ACS Appl. Mater. Interfaces 2010, 2, 1466-1470.

(25) Chu, W.; Zhang, Y.; Li, D.; Barrow, C. J.; Wang, H.; Yang, W. A Biomimetic Sensor for the Detection of Lead in Water. Biosens. Bioelectron. 2015, 67, 621-624.

(26) Huang, K.-W.; Yu, C.-J.; Tseng, W.-L. Sensitivity enhancement in the colorimetric detection of lead(II) ion using gallic acid-capped gold nanoparticles: Improving size distribution and minimizing interparticle repulsion. Biosens. Bioelectron. 2010, 25, 984-989.

(27) Yang, R.; Cheng, W.; Chen, X.; Qian, Q.; Zhang, Q.; Pan, Y.; Duan, P.; Miao, P. Color Space Transformation-Based Smartphone Algorithm for Colorimetric Urinalysis. ACS Omega 2018, 3, 1214112146

(28) Whitehead, H. D.; Waldman, J. V.; Wirth, D. M.; LeBlanc, G. 3D Printed UV-Visible Cuvette Adapter for Low-Cost and Versatile Spectroscopic Experiments. ACS Omega 2017, 2, 6118-6122.

(29) Shrivas, K.; Kant, T.; Karbhal, I.; Kurrey, R.; Sahu, B.; Sinha, D.; Patra, G. K.; Deb, M. K.; Pervez, S. Smartphone Coupled with Paper-Based Chemical Sensor for on-Site Determination of Iron (III) in Environmental and Biological Samples. Anal. Bioanal. Chem. 2020, 412, 1573-1583.

(30) Muhammad-aree, S.; Teepoo, S. On-Site Detection of Heavy Metals in Wastewater Using a Single Paper Strip Integrated with a Smartphone. Anal. Bioanal. Chem. 2020, 412, 1395-1405.

(31) Zhang, W.; Du, Q.; Dou, Z.; Ning, S.; Wang, Q.; Dong, Y.; Yue, Z.; Ye, W.; Liu, G. Ultrasensitive Detection of Lead (II) Ion by DarkField Spectroscopy and Glutathione Modified Gold Nanoparticles. Sens. Actuators, B 2020, 321, 128548.

(32) Yuan, J.; Christensen, P. R.; Wolf, M. O. Dynamic AntiCounterfeiting Security Features Using Multicolor Dianthryl Sulfoxides. Chem. Sci. 2019, 10, 10113-10121.

(33) Coleman, B.; Coarsey, C.; Kabir, M. A.; Asghar, W. Point-ofCare Colorimetric Analysis through Smartphone Video. Sens. Actuators, B 2019, 282, 225-231.

(34) Nguyen, V. D.; Van Nguyen, H.; Bui, K. H.; Seo, T. S. Smart Phone-Powered Capillary Electrophoresis on a Chip for Foodborne Bacteria Detection. Sens. Actuators, B 2019, 301, 127108.

(35) Li, S.; Lu, Y.; Liu, L.; Low, S. S.; Su, B.; Wu, J.; Zhu, L.; Li, C.; Liu, Q. Fingerprints Mapping and Biochemical Sensing on Smartphone by Electrochemiluminescence. Sens. Actuators, B 2019, 285, 34-41.

(36) Vietz, C.; Schütte, M. L.; Wei, Q.; Richter, L.; Lalkens, B.; Ozcan, A.; Tinnefeld, P.; Acuna, G. P. Benchmarking Smartphone Fluorescence-Based Microscopy with DNA Origami Nanobeads: Reducing the Gap toward Single-Molecule Sensitivity. ACS Omega 2019, 4, 637-642.

(37) Chung, S.; Breshears, L. E.; Perea, S.; Morrison, C. M.; Betancourt, W. Q.; Reynolds, K. A.; Yoon, J.-Y. Smartphone-Based Paper Microfluidic Particulometry of Norovirus from Environmental Water Samples at the Single Copy Level. ACS Omega 2019, 4, $11180-11188$.

(38) Wang, H.; Yang, L.; Chu, S.; Liu, B.; Zhang, Q.; Zou, L.; Yu, S.; Jiang, C. Semiquantitative Visual Detection of Lead Ions with a Smartphone via a Colorimetric Paper-Based Analytical Device. Anal. Chem. 2019, 91, 9292-9299.

(39) Nguyen, H.; Sung, Y.; O’Shaughnessy, K.; Shan, X.; Shih, W.-C. Smartphone Nanocolorimetry for On-Demand Lead Detection and Quantitation in Drinking Water. Anal. Chem. 2018, 90, 1151711522 .

(40) Sheregii, E.; Deręgowska, A.; Depciuch, J.; Wojnarowska, R.; Polit, J.; Broda, D.; Nechai, H.; Gonchar, M. Study of Optical Properties of a Glutathione Capped Gold Nanoparticles Using Linker (MHDA) by Fourier Transform Infra Red Spectroscopy and Surface Enhanced Raman Scattering. World Acad. Sci. Eng. Technol. 2013, 73, 611-614.

(41) Leff, D. V.; Brandt, L.; Heath, J. R. Synthesis and Characterization of Hydrophobic, Organically-Soluble Gold Nano- crystals Functionalized with Primary Amines. Langmuir 1996, 12, $4723-4730$

(42) Liu, C.-W.; Huang, C.-C.; Chang, H.-T. Highly Selective DNABased Sensor for Lead(II) and Mercury(II) Ions. Anal. Chem. 2009, $81,2383-2387$.

(43) Wang, Y.; Irudayaraj, J. A SERS DNAzyme Biosensor for Lead Ion Detection. Chem. Commun. 2011, 47, 4394-4396.

(44) Dasary, S. S. R.; Jones, Y. K.; Barnes, S. L.; Ray, P. C.; Singh, A. K. Alizarin Dye Based Ultrasensitive Plasmonic SERS Probe for Trace Level Cadmium Detection in Drinking Water. Sens. Actuators, B 2016, 224, 65-72.

(45) Botha, T. L.; James, T. E.; Wepener, V. Comparative Aquatic Toxicity of Gold Nanoparticles and Ionic Gold Using a Species Sensitivity Distribution Approach. J. Nanomater. 2015, 2015, 986902.

(46) Khan, N. A.; Niaz, A.; Zaman, M. I.; Khan, F. A.; Tariq, M. Sensitive and Selective Colorimetric Detection of $\mathrm{Pb}^{2+}$ by Silver Nanoparticles Synthesized from Aconitum Violaceum Plant Leaf Extract. Mater. Res. Bull. 2018, 102, 330-336.

(47) Tariq, J.; Zhang, Y.; Li, H.; Wen, Y.; Fan, X.; Lin, F.; Tan, L.; Yao, S. Ultrasensitive electrochemical aptasensor for thrombin based on the amplification of aptamer-AuNPs-HRP conjugates. Biosens. Bioelectron. 2011, 26, 2297-2303.

(48) Baldrich, E.; Restrepo, A.; O'Sullivan, C. K. Aptasensor Development: Elucidation of Critical Parameters for Optimal Aptamer Performance. Anal. Chem. 2004, 76, 7053-7063.

(49) Divsar, F.; Habibzadeh, K.; Shariati, S.; Shahriarinour, M. Aptamer Conjugated Silver Nanoparticles for the Colorimetric Detection of Arsenic Ions Using Response Surface Methodology. Anal. Methods 2015, 7, 4568-4576.

(50) Turkevich, J.; Stevenson, P. C.; Hillier, J. A Study of the Nucleation and Growth Processes in the Synthesis of Colloidal Gold. Discuss. Faraday Soc. 1951, 11, 55-75.

(51) Wheelus, C.; Najafabadi, M. M.; Khoshgoftaar, T. M.; Napolitano, A. Rudy Attack: Detection at the Network Level and Its Important Features. The Twenty-Ninth International Flairs Conference; AAAI Publications: Florida, 2016; pp 282-287.

(52) Ippoliti, P.; Nargi, F.; Han, J.; Casale, A.; Walsh, M.; Boettcher, T.; Dettman, J. Highly Accurate Classification of Biological Spores by Culture Medium for Forensic Attribution Using Multiple Chemical Signature Types and Machine Learning. Anal. Bioanal. Chem. 2020, 412, 4287-4299.

(53) Krizhevsky, A.; Sutskever, I.; Hinton, G. E. Imagenet Classification with Deep Convolutional Neural Networks. Advances in Neural Information Processing Systems; Association for Computing Machinery, 2017, pp 84-90.

(54) Hyndman, R. J.; Koehler, A. B. Another Look at Measures of Forecast Accuracy. Int. J. Forecast 2006, 22, 679-688. 